\title{
Break-Up of Aerosol Agglomerates in Highly Turbulent Gas Flow
}

\author{
Yasmine Ammar • Abdel Dehbi • Michael W. Reeks
}

Received: 5 May 2011 / Accepted: 9 May 2012 / Published online: 30 May 2012

(C) Springer Science+Business Media B.V. 2012

\begin{abstract}
Agglomerate aerosols in a turbulent flow may be subjected to very high turbulent shear rates which through the generation of lift and drag can overcome the adhesive forces binding the constituents of an agglomerate together and cause it to break-up. This paper presents an analysis of the experimental measurements of the breakup of agglomerates between $0.1-10 \mu \mathrm{m}$ in size, in a turbulent pipe flow followed by an expansion zone with a Reynolds numbers in the range $10^{5}$ to $10^{7}$. The analysis shows that even in wall bounded turbulence, the high turbulent shear stresses associated with the small scales of turbulence in the core can be the main source of breakup preceding any break-up that may occur by impaction at the wall. More importantly from these results, a computationally fast and efficient solution is obtained for the General Dynamic Equation (GDE) for agglomerate transport and breakup in highly turbulent flow. Furthermore the solution for the evolution of the aerosol size distribution is consistent with the experimental results. In the turbulent pipe flow section, the agglomerates are exposed continuously to turbulent shear stresses and experience more longer term breakup than in the expansion zone (following the pipe flow) where the exposure time is much less and break-up occurs instantaneously under the action of very high local turbulent shear stresses. The validity of certain approximations made in the model is considered. In particular, the inertia of the agglomerates characterised by a Stokes Number from 0.001 for the smallest particles up to 10 for $10 \mu \mathrm{m}$ particles and the fluctuations of the turbulent
\end{abstract}

\footnotetext{
Y. Ammar $(\bowtie) \cdot$ M. W. Reeks

School of Mechanical and Systems Engineering,

University of Newcastle upon Tyne,

Newcastle Upon Tyne NE1 7RU, UK

e-mail: Yasmine.ammar@ncl.ac.uk

Y. Ammar · A. Dehbi

Thermal-Hydraulics Laboratory,

Nuclear Energy and Safety Department,

Paul Scherrer Institute, 5232 Villigen PSI, Switzerland
} 
shear stresses are important physical phenomena which are not accounted for in the model.

Keywords Agglomerate·Turbulence ·Break-up·De-agglomeration • Aerosol

$\begin{array}{ll}\text { Abbreviations } \\ \text { BI } & \text { Berner Impactor } \\ \text { CFD } & \text { Computational Fluids Dynamics } \\ \text { CMD } & \text { Count Median Diameter } \\ \text { DNS } & \text { Direct Numerical Simulation } \\ \text { ELPI } & \text { Electrical Low Pressure Impactor } \\ \text { FBG } & \text { Fluidized bed generator } \\ \text { GDE } & \text { General Dynamic Equation } \\ \text { GSD } & \text { Geometric standard deviation } \\ \text { LDA } & \text { Laser Doppler anemometer } \\ \text { OPC } & \text { Optical Particle Counter } \\ \text { PSD } & \text { Particle size distribution } \\ \text { SEM } & \text { Scanning electron microscopy }\end{array}$

\section{Introduction}

In turbulent flow, individual aerosol agglomerates interact with turbulent structures. In particular, they are segregated into straining regions where they either break up through the high shearing of the flow in these regions or continue to grow with enhanced collision rates [1]. For the most part in clouds for instance, there is no breakup, and droplets will continue to grow at an enhanced rate until they precipitate out (rain) due to gravitational settling. In other situations where solid agglomerates are formed, break up might be due to either inter-agglomerate collisions, through impact with a wall, or aerodynamic shear forces as in a turbulent jet. For instance, chemical engineers actively investigate the competition between agglomeration and break-up in bioreactors [2,3]. If the agglomerate suspension is dilute enough, breakup becomes the predominant process as the agglomeration rate becomes negligible compared to break-up rate.

The break-up of aerosol agglomerates in turbulent flow has application in a number of important environmental and industrial processes. Of particular importance is the release of radioactive aerosols in a severe nuclear accident. The evidence suggests strongly that break-up could play a role in reducing the radioactive aerosol size distribution and increasing the rate of release to the environment in the event of a severe accident. Of similar importance are the size and shape of agglomerates in the pharmaceutical, detergent and food processing industries where agglomerates offer better 'flowability' with less dust formation in comparison with fine powders [4].

Break-up mechanisms have been extensively studied and understood in the context of break-up of droplets in turbulent flows [5] because the mechanisms involving droplet deformation are well-known. However the break-up of solid agglomerates presents more uncertainties mainly due to the difficulty of expressing the adhesive forces for complex agglomerate morphology. 
The study presented here is concerned with providing a model for the breakup mechanism for aerosol agglomerates smaller than the Kolmogorov scale of turbulent gas flow at very high Reynolds number (typically $10^{5}$ and above). Breakup mechanisms due to both wall bounded and free turbulent shear stresses on aerosol particles are assessed. The timescales of the physical phenomena applied to highly turbulent flow laden particles are compared, based on an experimental investigation described in $[6,7]$. The experiments consisted of measuring the size of the agglomerates by light scattering at the inlet and the outlet of a test section composed of a tube of varying length $(20 \mathrm{~cm}, 2 \mathrm{~m}$ and $4 \mathrm{~m}$ long pipes were used) and an expansion zone. Possible dependency of the de-agglomeration on the level of turbulence was investigated by varying the velocity at the inlet of the test section; this velocity ranged from a few $\mathrm{m} / \mathrm{s}$ all the way to $\sim 150 \mathrm{~m} / \mathrm{s}$.

As originally suggested by Sonntag and Russel [8], break-up was assumed to be instantaneous with respect to the smallest time scale of turbulence and based on this assumption, it was shown that the break-up event takes place before the agglomerate impacts to the wall. Aerosol agglomerates refer here to an assembly of nanometre solid primary particles with branch-like and irregular structures characterized by self similarity over several orders of magnitude [9]. In this paper, it is assumed that even if the agglomerates are composed of primary particles with varying shape and diameter, they consist of identical spherical primary particles held together by adhesive forces.

This paper first presents the state of the art of aerosol break-up modelling. The Computational Fluids Dynamics (CFD) predictions are validated against experimental measurements and the relevant parameters responsible for break-up are determined by comparing the timescales of different physical phenomena. The evolution of the particle size distribution in the geometry is finally obtained by solving the General Dynamic Equation (GDE) (also known as population balance equation). The implementation is performed along the turbulent flow streamlines for inertialess aerosol particles and the applicability of the results is discussed with regards to the assumptions made in the model.

\section{Break-Up Modelling: State of the Art}

Regardless of the physical phenomena involved, two pieces of information are required in order to quantify the break-up: (i) the rate at which the break-up occurs or particle break-up rate $a^{\prime}$ and, (ii) the number and sizes of fragment particles which is given by the break-up fragment distribution function $b^{\prime}(L, l)$ for an agglomerate of size $l$ breaking up into fragments of characteristic size $L$ defined as $\mathrm{L}^{3}=v$ with $v$ the particle volume. Both are accounted for in a simplified form of the GDE for break-up [10]:

$$
\begin{aligned}
& \frac{\partial n(L, t)}{\partial t}+\mathbf{U}_{\mathbf{f}} \cdot \nabla n(L, t)-\nabla\left(D_{T} \nabla n(L, t)\right) \\
& =\int_{L}^{\infty} a^{\prime}(l, t) b^{\prime}(L, l) n(l, t) d l-a^{\prime}(L, t) n(L, t)
\end{aligned}
$$

Here $n(L, t)$ is the Particle Size Distribution (PSD), $t$ is the time, $\mathbf{U}_{\mathrm{f}}$ is the mean fluid velocity and $D_{T}$ is the turbulent diffusivity. The first term on the right hand side is 
the rate of birth of particles of size $L$, due to the break-up of particles of size $l$ bigger than $L$ whereas the second term is the rate of death of particles of size $L$ due to the break-up of particles of size $L$.

Existing models for the break-up rate and fragment distributions will be reviewed in Sections 2.1 and 2.2 respectively. Section 2.3 will review the existing methods to solve Eq. 1.

\subsection{Expression for the break-up rate}

The expression for particle break-up rate often contains parameters which need to be deduced or inferred from experimental data. Kramer and Clark [11] derived an expression for the break-up rate based on flow-induced normal stresses for solid particles and similar kernels have been developed for droplet and bubble break-up in turbulent dispersions $[12,13]$.

For agglomerates smaller than the Kolmogorov length scale, the hydrodynamic stress is determined by the viscosity and local dissipation rate $\varepsilon$ whose fluctuations are highly intermittent. Kusters [2] gave an expression for the break-up rate of such agglomerates in homogeneous turbulent and the local dissipation rate flow assuming a Gaussian distribution for the local dissipation rate fluctuations, namely:

$$
a^{\prime}(\varepsilon, L)=\left(\frac{4}{15 \pi}\right)^{1 / 2}\left(\frac{\langle\varepsilon\rangle}{v}\right)^{-1 / 2} \exp \left(-\frac{\varepsilon^{b}(L)}{\langle\varepsilon\rangle}\right),
$$

where $\varepsilon_{b}$ is the critical turbulent energy dissipation rate above which the break-up occurs. It is related to characteristic agglomerate size $L$ such as:

$$
\varepsilon_{b}(L)=P[L]^{-1 / q},
$$

where $P$ and $q$ are parameters depending on the response of the agglomerate to turbulent stresses. Equation 3 is consistent with the fact that $\varepsilon_{b}$ decreases with increasing agglomerate size since larger particles are more susceptible to break up than smaller ones. This fact was observed in both experimental [14] and simulation investigations [15], the parameter $q$ varying between 0.4 and 5 for various flows and agglomerate sizes. The break-up rate can also be described by a power law such as:

$$
a^{\prime}(\varepsilon, L)=P_{1}\left(\frac{\varepsilon}{v}\right)^{P_{2}} L^{P_{3}}
$$

$P_{1}, P_{2}$ and $P_{3}$ are empirical parameters that depend on the flow and the agglomerate type under investigation.

Babler et al. [16] discussed Eqs. 2 and 4. In particular, they pointed out that unlike experimental and theoretical evidences, these equations fail to include the existence of a critical agglomerate size below which no break-up is observed. They proposed a power-law expression to correlate the critical magnitude of homogeneous turbulence fluctuations that causes break-up with the agglomerate properties and similar to Eq. 3. The agglomerates were assumed to break up instantaneously compared to the Kolmogorov time scale. The applicability of this assumption was discussed in this paper for agglomerates with characteristic response time smaller than the Kolmogorov time scale. An expression for the break-up rate was derived 
for a Gaussian turbulence fluctuation distribution. They showed that it reduces to an exponential function and a power law similar to Eqs. 2 and 4 in the limits of very small and very large agglomerates respectively.

\subsection{The fragment distribution}

It is commonly assumed that the break-up of one agglomerate produces two fragments of equal size $[17,18]$. Erosion [19] is another break-up model referring to the break-up of one parent particle into one very small particle and another with size close to the original. However, the break-up of one fractal agglomerate in turbulent flow could result into 3 or 4 smaller sizes as illustrated by Monte Carlo simulations of aggregate break-up in shear flow [15]. These fragments were denser than the parent agglomerate and no direct relationship was found between parent and daughter structure. A major problem with this kind of applications is dealing with the statistics associated with both turbulence and fractal agglomerates.

The literature contains various standard fragment size distributions such as a normal [13, 20] or bimodal distribution for droplets [21, 22] and a lognormal [23] distribution for flocs in a flowing suspension. The $U$ shaped fragment distribution is also widely used. It represents processes in which the most probable break-up results in small and large fragments with zero probability of equal-sized fragments [24-26]. A generalized treatment has been proposed by Kostoglou et al. [27] under the form of a normal distribution. Diemer and Olson [28] provided a sufficiently flexible form so that a weighted sum of power law product terms describes most of the fragment distributions discussed above.

\subsection{How to solve the break-up equation}

The break-up equation given by Eq. 1 needs to be solved. In the case where the system remains fully mixed throughout the break-up process and the local time derivative for the PSD is the only non negligible term on the Left Hand Side of Eq. 1, the modelling of the break-up rate by a power-law in particle size and with self similar daughters, yields similarity solutions (often in the form of modified gamma functions) for a broad class of break-up problems [29-32]. But outside these simplifying assumptions, no general analytic solution exists. One successful approach is based on Monte Carlo methods for the population of particles undergoing a birth-death process [33]. For more details of the treatment of GDE equations see Ramkrishna [10].

The method for solving the GDE equation needs to be computationally efficient whilst being able to describe the particle size distribution accurately. The GDE can be discretised in particle size using a number of different methods [34-36] but it requires a relatively large number of particle size classes which renders the resolution of this equation virtually impossible for CFD code since the solution of these classes has to be calculated in each cell of the computational domain resulting in a very high computation time and extensive memory requirements. Nevertheless, calculation of the moments of the particle size distribution is often sufficient for comparison with experiments as the instruments only measure average quantities. 
The $k-$ th moment of the PSD is defined as:

$$
m_{k}(\mathbf{x}, t)=\int_{0}^{+\infty} n(v, \mathbf{x}, t) v^{k} d v=\int_{0}^{+\infty} n(l, \mathbf{x}, t) l^{3 k} d l,
$$

Where $n(v, \mathbf{x}, t)$ is the distribution function for the number density of particles having volumes in the range $v$ to $v+d v$.

If the particle size is characterized by its volume, the zeroth moment $m_{0}$ is the total particle number density and the first moment $m_{1}$ is the particle volume fraction at a given location. For the specific case where particle material density remains constant, $m_{1}$ is conserved in a closed system since the invariance of the first moment refers to the conservation of mass.

However, except for special restrictive forms, solving the set of transport equations for moments requires closure models for the missing moments. As was first shown by McGraw [37] in describing the size dependent growth in aerosols, this closure problem can alternatively be overcome by invoking a quadrature approximation for the moments referred to as to the Quadrature Method Of Moments (QMOM). The method gives in some cases better results than those based on pre-assumed particle size distribution forms and the alternative closure technique described by a Laguerre series expansion on the gamma distribution. Marchisio et al. [3] validated the method for size-dependent growth and aggregation against both Monte Carlo simulations and analytical solutions using several functional forms for the agglomeration, and showed good agreement of the results with those obtained using a discretised approach.

The special case where diffusive transport can be neglected with respect to the convective transport is of particular relevance for very high Reynolds number pipe flows. Using this condition, it becomes possible to perform the integration of the GDE along a streamline which significantly reduces the computational time and as a consequence allows one to consider the direct discretisation of the Population Balance equations into sections. Various authors have combined the Eulerian description of the carrier flow with a Lagrangian description of the GDE to determine the droplet size distribution in air-water flow [38-43]. In the particular case of zero diffusion, the distribution evolution in the particle state space is thus obtained without utilising any closure approximation.

\section{Turbulent Gas Flow}

The flow is based on the experimental setting described in [6, 7].

The test facility is depicted in Fig. 1. It consists of a Fluidized Bed Generator (FBG) a mixing chamber where $\mathrm{TiO}_{2}$ aerosol coming from the FBG is mixed with the main air flow and the test tube followed by an expansion zone. The instrumentation consists of two Optical Particle Counters (OPCs) at the inlet and outlet of the tube, an Electrical Low Pressure Impactor (ELPI), a membrane filter, 4 Berner Impactors (BIs) and a sampling system to extract, when required, aerosols from the gas stream for subsequent morphological analysis by means of a scanning electron microscope (SEM). The test section is composed of 3 sections: a smooth contraction, a $16.9 \mathrm{~mm}$ diameter straight tube of variable length and a conical expansion zone of $140 \mathrm{~mm}$ 


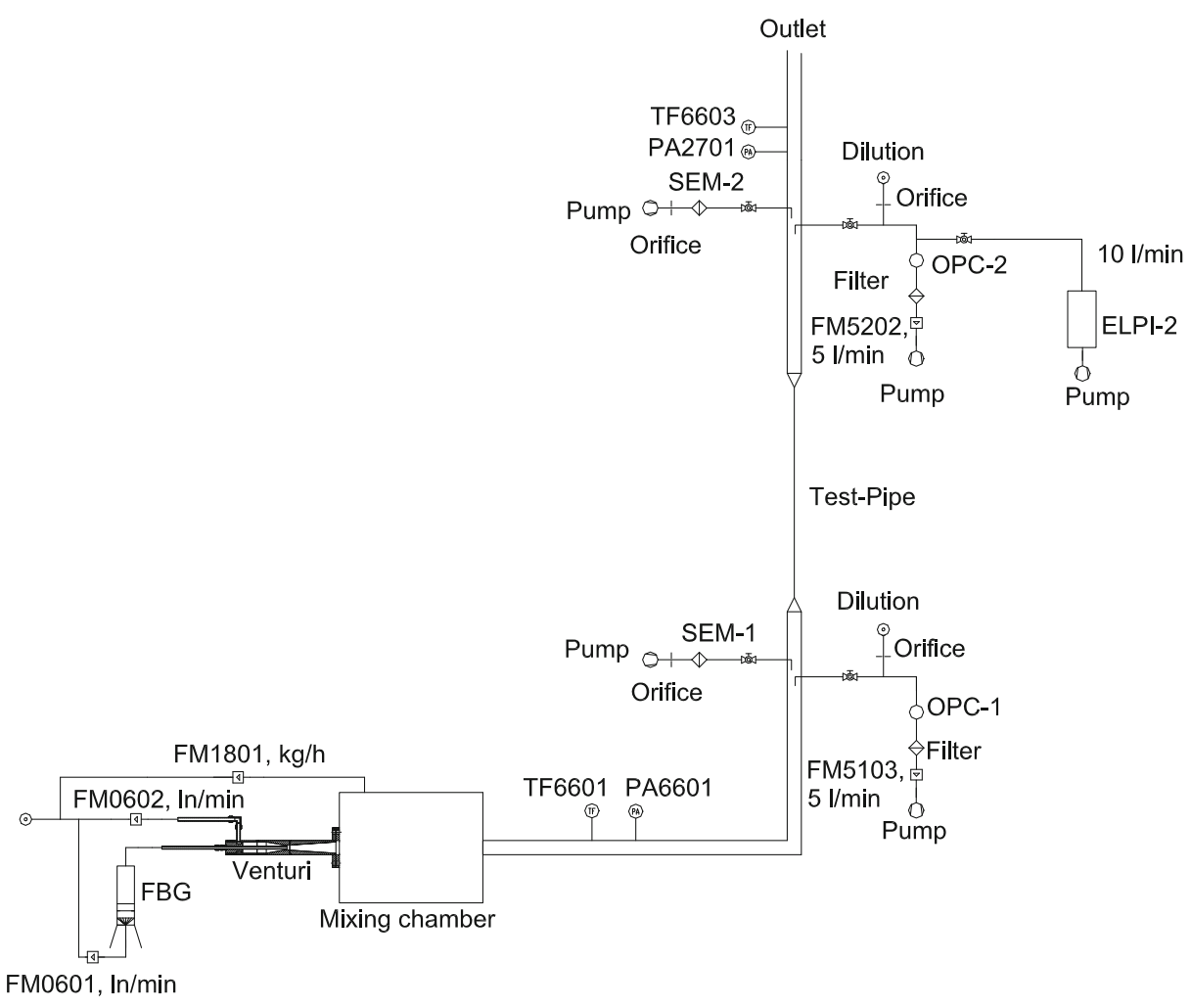

Fig. 1 Schematic representation of the experimental setting

diameter (cf. Fig. 2). The inlet composed of three parts, two conical contractions separated by a $152 \mathrm{~mm}$ long tube, describes a smooth diameter reduction from $70.9 \mathrm{~mm}$ to $16.8 \mathrm{~mm}$ which avoids break-up to occur prior to the tube. Due to high velocity inside the straight tube, measurements and the aerosol samples cannot be taken inside the straight tube section directly but prior and after, inside the contraction inlet and expansion outlet respectively.

The break-up of particle agglomerates has been studied in the literature for Reynolds numbers in the range from 5000 to 50000 [44, 45]. The current study extends this range up to Reynolds numbers of $10^{5}-10^{7}$ for solid gas compressible turbulent flows. The next section will discuss the modelling of turbulent flows at this Re range.

For highly turbulent flows, only Reynolds Averaged Navier Stokes equations which model the entire spectrum of the turbulent motions can be solved although the performance of this approach is uneven in flows outside of the calibration range of the models.

CFD calculations have been performed for mass flow rates in the range $20 \mathrm{~kg} / \mathrm{h}-$ $450 \mathrm{~kg} / \mathrm{h}$ using the commercial package FLUENT 6.3 and accompanied by LDA velocity measurements in an air jet for 2 mass flow rates, $110 \mathrm{~kg} / \mathrm{h}$ and $450 \mathrm{~kg} / \mathrm{h}$. A 2D axis-symmetric configuration and the standard k-epsilon turbulent model for compressible flow were used in every CFD simulation. For more details, see [6]. 


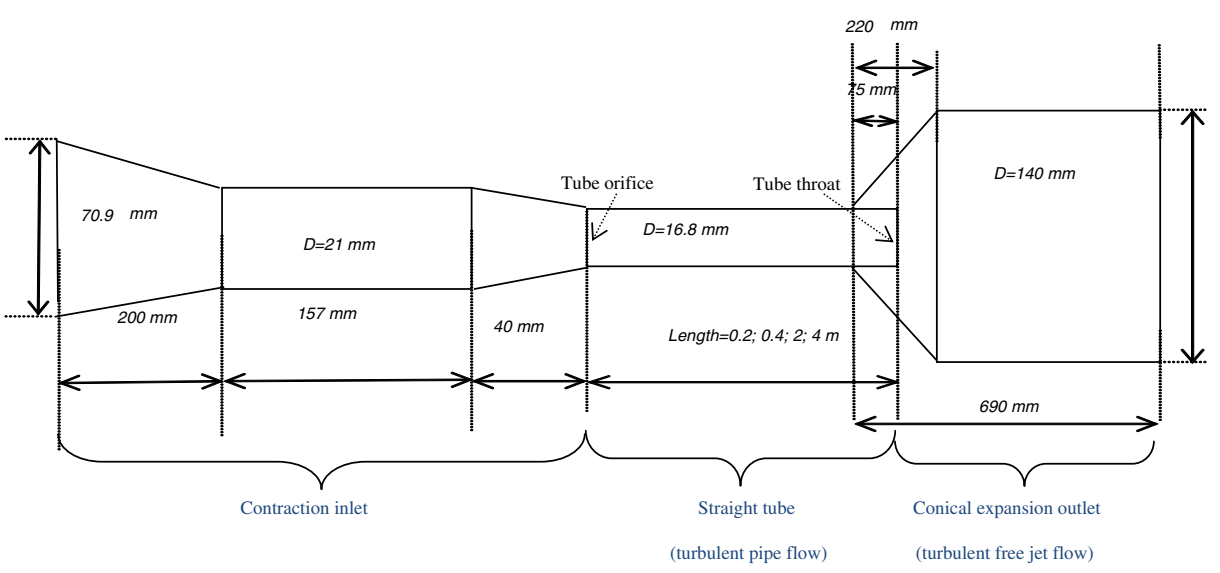

Fig. 2 Schematic representation of the experimental setting

The flow in the boundary layer was calculated using wall enhanced treatment with the nearest wall cell $y^{+}$close to 1 . The calculations were conducted until scaled residuals dropped below $10^{-6}$ and the mean wall $y^{+}$and shear wall stresses reached stationary values.

Gauge pressure was measured along the pipe having a length of $4 \mathrm{~m}$, Fig. 3, for a gas mass flow rate of $370 \mathrm{~kg} / \mathrm{h}$. The CFD calculations predicted very well the experimental trend.

The velocities were measured with a Laser Doppler Anemometer (LDA) at different downstream distances for two gas mass flow rates of $110 \mathrm{~kg} / \mathrm{h}$ and $450 \mathrm{~kg} / \mathrm{h}$ in a vertical jet located in the $690 \mathrm{~mm}$ long conical expansion (cf. Fig. 2), emerging from a straight tube with a length of $400 \mathrm{~mm}$. LDA measurements and comparison with CFD calculations are described in details in [6]. A very good agreement could be

Fig. 3 Gauge pressure profile measured along the streamwise direction for a mass flow rate of $370 \mathrm{~kg} / \mathrm{h}$ : comparison with FLUENT simulation results

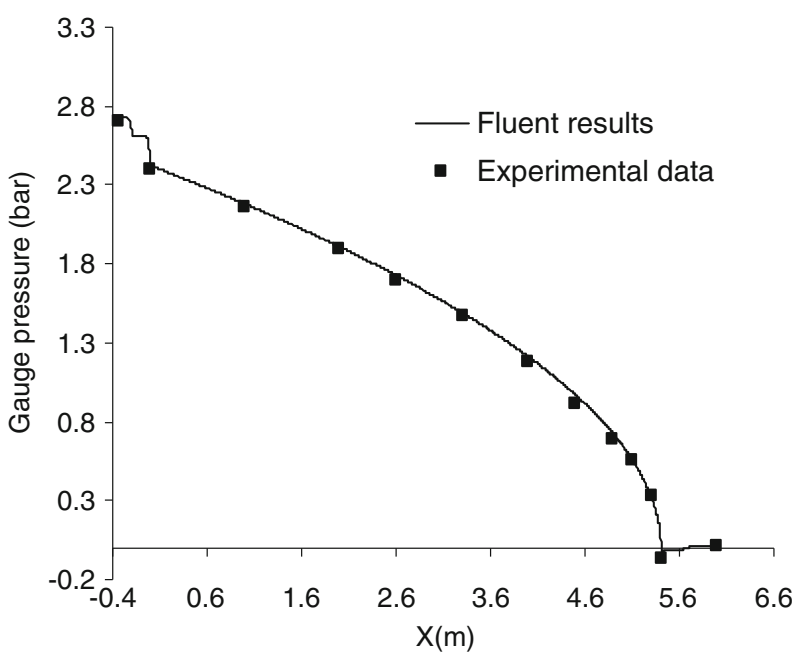


established between the LDA measurements and the CFD calculations with respect to the velocity and the turbulent kinetic energy measurements. Therefore, in the subsequent sections, the necessary turbulent quantities can be extracted from the CFD calculations.

\section{Identification of the Mechanisms Responsible for Break-Up}

The aerosol agglomerate size considered here is in the range $0.1 \mu \mathrm{m}-5 \mu \mathrm{m}$. Various transport mechanisms and forces are applied to aerosols in highly turbulent flows. The objective of this section is to demonstrate the relative importance of break-up and to identify the forces causing the agglomerates to break-up.

\subsection{Particle concentration across the pipe section}

Particle-tracking simulations for a $2 \mathrm{~m}$ straight tube section as shown on Fig. 2 were carried out using Fluent 6.3 by injecting a monodisperse sample of 1000 particles with a diameter of one micron, homogeneously distributed across the inlet pipe section. Inter-particle collision was not taken into account. The turbulent dispersion model based on the Langevin equation was used as first introduced by Iliopoulos and Hanratty [46] and more recently by Dehbi [47]. This model takes into account the anisotropy of turbulence in the boundary layer. The walls were modelled as perfectly reflecting [7].

The number density in each annular shell, normalized by the average number density in the whole domain, is presented as a function of the normalised radial position from the centre of the annular shells for different time steps in Fig. 4. The time $t=1$ corresponds to the transit time for particles to travel the pipe length. The pipe wall is located at a radial position of 1 . The particles were injected uniformly which means that the normalised number density in each annulus was one to start with. The particle concentration remained quasi-uniform in the pipe section except for the last annular shells close to the wall where the particle number density is

Fig. 4 Number density versus radial position for different times $\mathrm{t}$

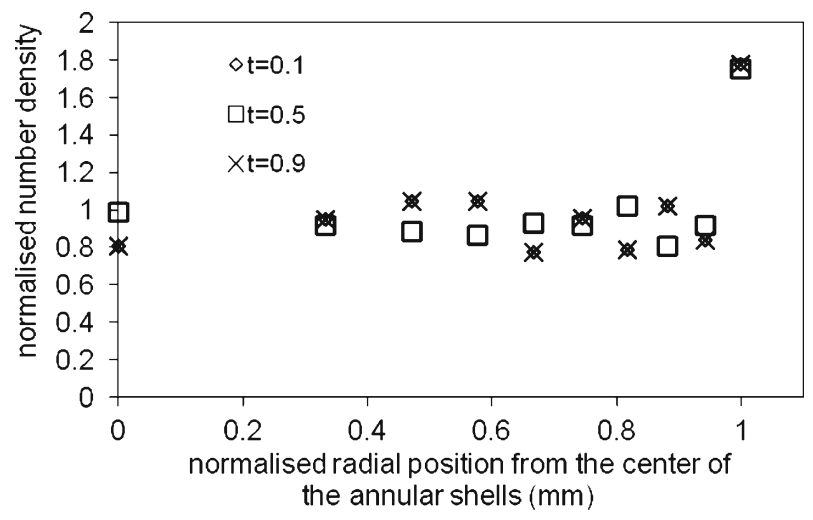


almost equal to twice the value in the other shells. This latter phenomenon refers to preferential concentration of inertial particles in turbulent boundary layers identified by [48].

\subsection{Relative importance of break-up}

In the bulk, both agglomeration and break-up can typically take place. The characteristic time for agglomeration is defined as [49]:

$$
\tau_{A}=\frac{1}{k^{A} N_{t}}
$$

where $k^{A}$ is the collision kernel and $N_{t}$ is the volume-averaged total number density of agglomerates at steady state determined experimentally.

The collision rate is typically between the limits for larger particles given by Abrahamson [50] and for smaller particles given by Saffman and Turner [51]. Saffman and Turner defined the collision kernel between two particles of radius $R_{i}$ and $R_{j}$ such that:

$$
k^{A}(i, j)=1.294\left(\frac{\varepsilon}{v}\right)^{1 / 2}\left(R_{i}+R_{j}\right)^{3}
$$

Abrahamson gives the collision kernel between two particles of radius $R_{i}$ and $R_{j}$ and RMS velocity $v_{i}$ and $v_{j}$ :

$$
k^{A}(i, j)=0.5\left(R_{i}+R_{j}\right)^{2}\left[\frac{16 \pi}{3}\left(v_{i}^{2}+v_{j}^{2}\right)\right]^{1 / 2},
$$

where $v_{i}=u_{f}\left(1+1.5 \frac{\tau_{p_{i} \varepsilon}}{u_{f}^{2}}\right)^{-1 / 2}$

$u_{f}$ is the RMS of the fluid velocity and the particle response time is defined as:

$$
\tau_{p_{i}}=\frac{4 \rho_{p} R_{i}^{2}}{18 \mu}
$$

The characteristic time for break-up is defined as:

$$
\tau_{B}=\frac{1}{k^{B}},
$$

where $k^{B}$ is the break-up rate.

The break-up rate cannot exceed the characteristic frequency for the smallest (Kolmogorov) scale of turbulence $\tau_{K}$ defined as:

$$
k_{\max }^{B}=\tau_{K}^{-1}=\left(\frac{4}{15 \pi}\right)^{1 / 2}\left(\frac{\varepsilon}{v}\right)^{1 / 2}
$$

In order to determine the break-up rate, the dissipation rate of turbulent kinetic energy needs to be estimated. Here, CFD simulation is not used to calculate the turbulent kinetic energy. This illustrates a methodology in aerosol physics problems which consists in preliminary calculations for the identification of the physical 
phenomena which need to be modelled. Far from the pipe wall, in the inertial sublayer, experiments have shown that the dissipation rate of turbulence kinetic energy in pipe flow can be expressed as [52]:

$$
\varepsilon=\frac{u^{* 3}}{\kappa D}
$$

The friction velocity for normalization is evaluated from Blasius formula

$$
u^{*}=\left[0.03955 \mathrm{Re}^{-0.25}\right]^{0.5} U_{m},
$$

with $U_{m}$ being the bulk mean velocity and $\kappa$ the Von Karman constant which, for large Reynolds numbers, tends to $1 / 3$.

Near the pipe wall, the dimensionless dissipation rate for the turbulent kinetic energy is curve-fitted to the Direct Numerical Simulation (DNS) data of a twodimensional channel flow of Mansour used by Dreeben and Pope [53] such that

$$
\varepsilon^{+}=\frac{1}{4.529+0.0116\left(y^{+}\right)^{1.75}+0.768\left(y^{+}\right)^{0.5}}
$$

$y^{+}=\frac{y u^{*}}{v}$ is the wall unit away from the pipe wall.

The dissipation rate of turbulence kinetic energy is then given by:

$$
\varepsilon=\frac{u^{* 4}}{v} \varepsilon^{+}
$$

The expression for the dissipation rate for the turbulent kinetic energy is accurate only for turbulent channel and pipe flows of Reynolds numbers comparable to that of the $D N S\left(\mathrm{Re} \cong 10^{4}\right)$. However, it is shown that the profile near the wall $\left(y^{+}<50\right)$ is relatively insensitive to the change of Reynolds number [54].

For instance, given a bulk velocity of $200 \mathrm{~m} / \mathrm{s}$ inside a pipe, the dissipation rate amounts to $\varepsilon \cong 10^{5} \mathrm{~m}^{2} / \mathrm{s}^{3}$ in the bulk of the flow using Eq. 11 and $\varepsilon \cong 10^{7} \mathrm{~m}^{2} / \mathrm{s}^{3}$ in the boundary layer using Eq. $14\left(1<y^{+}<50\right)$. Equations 6 and 9 can then be used to estimate the range of values taken by the ratio of the characteristic time for agglomeration $\tau_{A}$ over the characteristic time for break-up $\tau_{B}$. With reference to Table 1 , one obtains $\frac{\tau_{A}}{\tau_{B}}$ in the range $20-10^{10}$ which indicates that the break-up is a much faster process than agglomeration. Hence we can subsequently neglect agglomeration in front of break-up in this study.

Table 1 Values of the parameters for the agglomeration and break-up characteristic time calculation

\begin{tabular}{ll}
\hline Particle size $(\mu \mathrm{m})$ & $0.1-1$ \\
Particle density $\left(\mathrm{kg} / \mathrm{m}^{3}\right)$ & $10^{3}$ \\
Maximum volume-averaged total number & $3.10^{9}$ \\
$\quad$ density of agglomerates measured in the & \\
$\quad$ experiments $\left(\# / \mathrm{m}^{3}\right)$ & \\
Bulk mean velocity $(\mathrm{m} / \mathrm{s})$ & 200 \\
$\quad \begin{array}{l}\text { Dissipation rate of turbulence } \\
\text { kinetic energy }\left(\mathrm{m}^{2} / \mathrm{s}^{3}\right)\end{array}$ & $10^{5}-10^{7}$ \\
$\quad \begin{array}{l}\text { Characteristic time for break-up (s) } \\
\text { Characteristic time for Turbulent }\end{array}$ & $3.96 \cdot 10^{-6}-1.25 \cdot 10^{-5}$ \\
$\quad$ agglomeration described by Eq. $7(\mathrm{~s})$ & $10^{-2}-10^{4}$ \\
$\quad \begin{array}{l}\text { Characteristic time for kinetic } \\
\text { agglomeration described by Eq. } 8(\mathrm{~s})\end{array}$ & $10^{-4}-10^{2}$ \\
\hline
\end{tabular}




\subsection{Break-up mechanisms}

Wengeler and Nirschl [44] showed that the elongational and turbulent stresses were crucial for nanometer particle break-up. The elongational stresses are proportional to the mean shear rate $\overline{\dot{\gamma}}_{m}=\sqrt{E / v}$ with $E$ the dissipation rate of the mean flow and the turbulent stresses are proportional to the turbulent shear rate $\bar{\gamma}=\sqrt{\varepsilon / \nu}$. But comparing the value of the mean shear rate with the turbulent strain rate along the streamwise direction within FLUENT (cf. Fig. 5), it can be seen that under the investigated conditions, turbulence is clearly the dominant mechanism within the range of Reynolds number considered in this work and therefore, only turbulent stresses will be considered subsequently.

The size of the smallest eddies corresponding to the Kolmogorov length scale in the turbulent jet reaches a minimum after the throat. Figure 6 gives the value of the minimum Kolmogorov length scale computed within FLUENT as a function of the Reynolds numbers. The minimum Kolmogorov length scale is obtained a few centimetres after the throat. It is worth mentioning that the curve shown in Fig. 6 was drawn by increasing the inlet mass flow rates from 20 to $450 \mathrm{~kg} / \mathrm{h}$ and by calculating, for each mass flow rate, the value of the minimum Kolmogorov scale and the corresponding Reynolds number. We can see that the values of the Kolmogorov length scale in this region lie in the range $\sim 4-10 \mu \mathrm{m}$. This is larger than the particle sizes with Count Mean Diameter (CMD) $0.6 \mu \mathrm{m}$ (Geometric Standard Deviation (GSD) 2) measured at the outlet of the tube and $2.5 \mu \mathrm{m}$ (GSD 3 respectively) measured at the inlet of the tube. This confirms that the turbulent forces causing the agglomerates to break-up are in the viscous subrange of turbulence.

Sonic conditions are reached in the experiments. The elapsed time for particles to pass through the shock is approximated to the ratio of the particle size over the sound speed [55]. As shown in Fig. 7, the Mach number reaches 1.2 at the throat for a velocity of $350 \mathrm{~m} / \mathrm{s}$ which corresponds to a maximum sound speed of approximately $291.6 \mathrm{~m} / \mathrm{s}$. For information, the magnitude of the dissipation rate for turbulent kinetic energy is given in Fig. 8 at the same location. The corresponding elapsed time magnitude is approximately $10^{-10} \mathrm{~s}$ for $0.1 \mu \mathrm{m}$ particle while it is approximately $10^{-8} \mathrm{~s}$ for $10 \mu \mathrm{m}$ particle. Supposing that the break-up rate due to the shock cannot exceed

Fig. 5 Comparison of the mean elongational rate with the turbulent shear rate in the $4 \mathrm{~m}$ tube versus Re

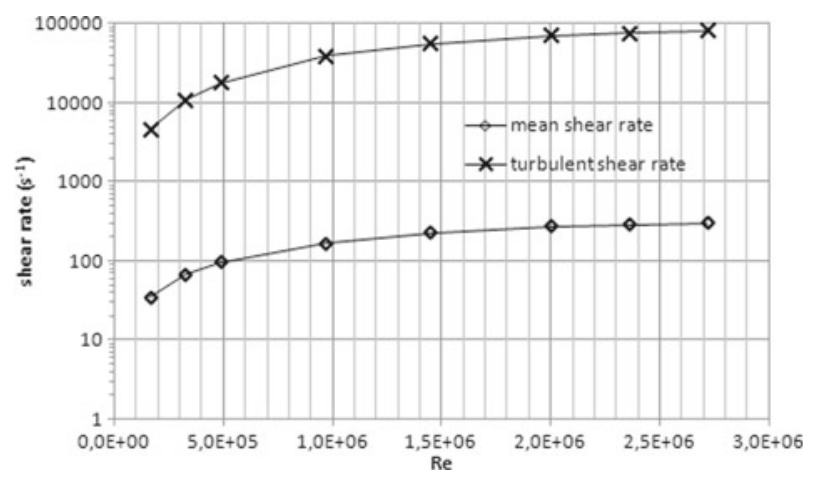


Fig. 6 Minimum Kolmogorov length scale against the level of turbulence for $20 \mathrm{~cm}$ tube

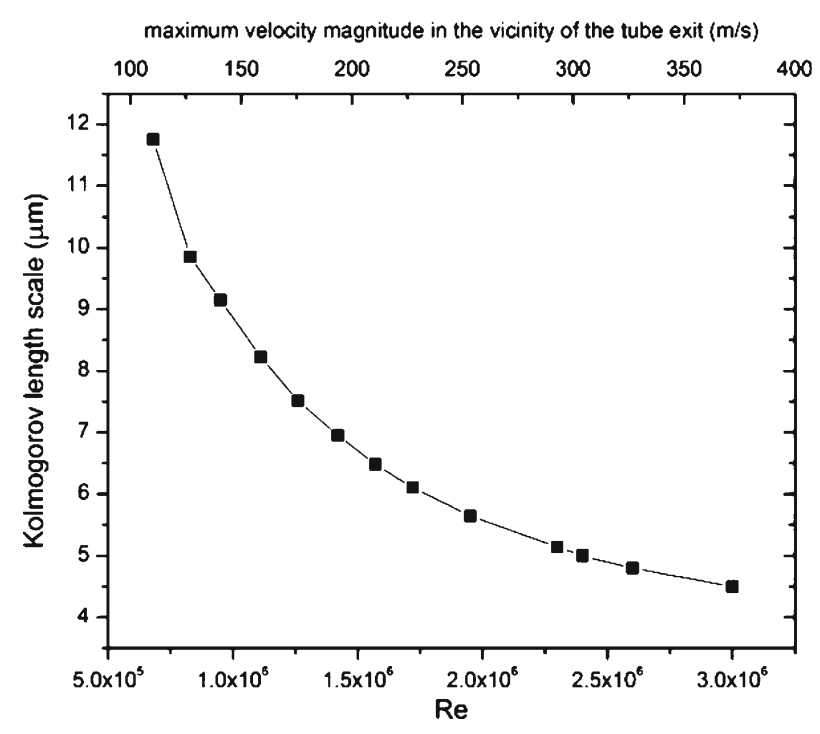

In the pipe
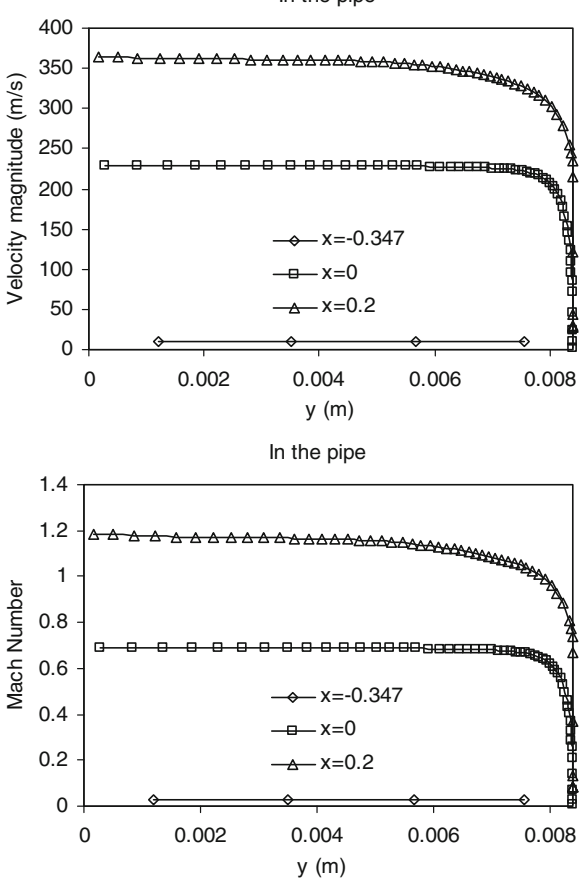

In the expansion zone

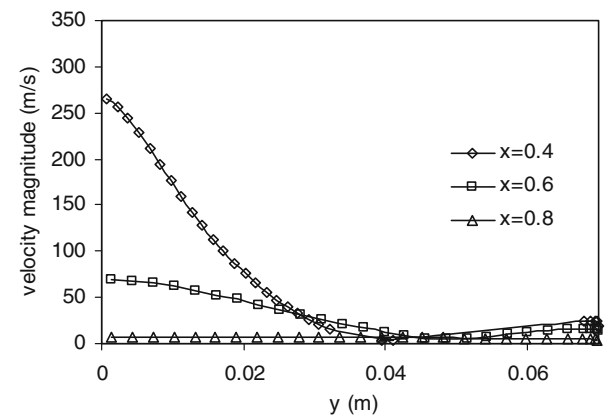

In the expansion zone

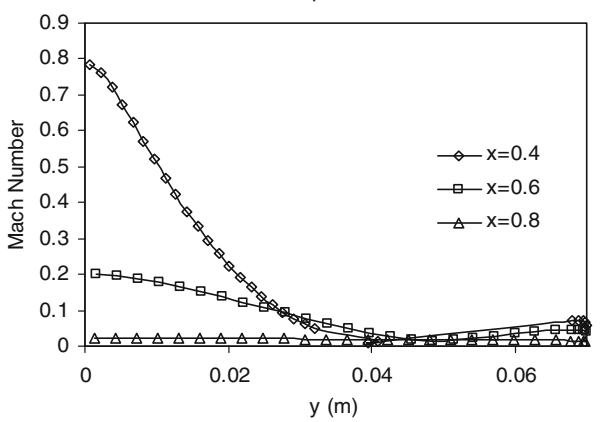

Fig. 7 Mach number and velocity magnitude versus the normal direction y to the pipe axis for different positions $\mathrm{x}$ along the streamwise direction. The tube orifice is located at $\mathrm{x}=0$ and the tube throat at $\mathrm{x}=0.2 \mathrm{~m}$ 

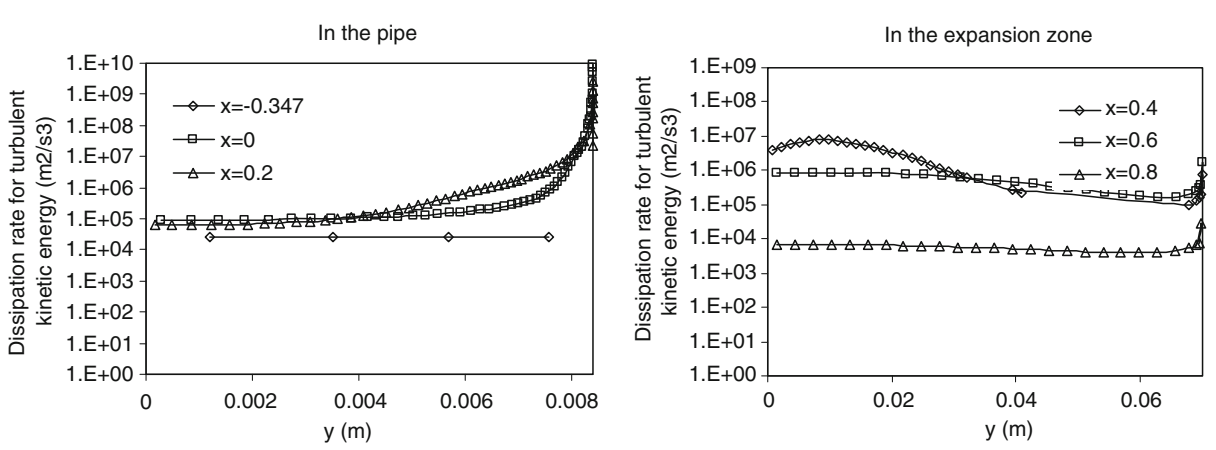

Fig. 8 Dissipation rate for turbulent kinetic energy versus the normal direction $y$ to the pipe axis for different positions $\mathrm{x}$ along the streamwise direction. The tube orifice is located at $\mathrm{x}=0$ and the tube throat at $\mathrm{x}=0.2 \mathrm{~m}$

the sound wave frequency of the order of $c / D \sim 1.74 .10^{4} \mathrm{~s}^{-1}$ with $D$ the diameter of the tube, the particles going through the shock are more likely to break-up due to turbulence with a rate of the order of the Kolmogorov rate $\sim 10^{6} \mathrm{~s}^{-1}$. It is therefore assumed that the break-up due to the shock can be neglected.

The evidence would suggest therefore that there is no extra force acting on the agglomerates apart from that due to the turbulent shear stresses.

At the wall, impaction may play a role. The impaction rate refers to the rate at which particles collide with the pipe surface area. It can be expressed as:

$$
\eta_{I}=\frac{\left|\mathbf{v}_{I}\right| \pi D L}{\left(\pi D^{2} / 4\right) L}=\frac{4\left|\mathbf{v}_{I}\right|}{D}
$$

where $\mathbf{v}_{I}$ is the impaction velocity, $D$ is the diameter of the pipe $(16.8 \mathrm{~mm}), L$ is the length of the pipe.

Figure 9 gives the cumulative distribution of impaction velocity normal to the wall determined from tracking 1000 particles up to the wall and computing their velocities

Fig. 9 Cumulative distribution of the impaction velocity normal to the wall calculated with particle tracking within FLUENT 6.3

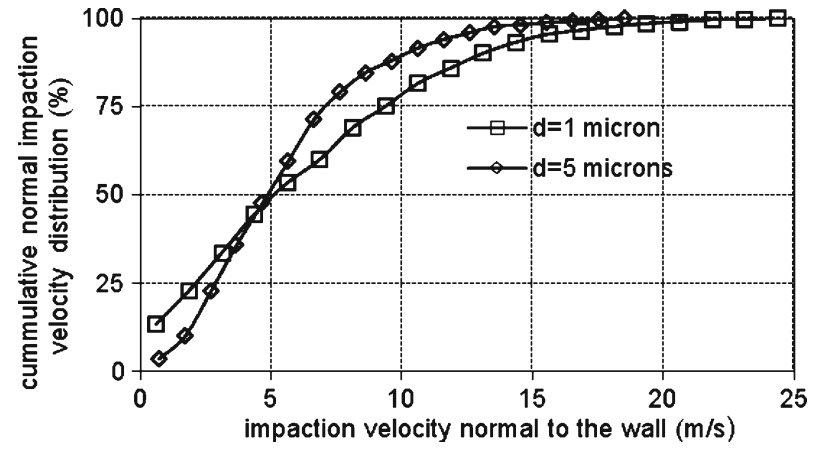


at the point of impaction within FLUENT 6.3. 50\% of particles have an impaction velocity less than $5 \mathrm{~m} / \mathrm{s}$ for both $1 \mu \mathrm{m}$ and $5 \mu \mathrm{m}$ particle geometrical sizes.

It is assumed that the break-up rate due to turbulence cannot exceed the Kolmogorov rate while the break-up due to impaction at the wall cannot exceed the rate at which particles collide at the pipe wall. Therefore, in order to identify the relevant break-up mechanisms taken place at the pipe wall, one needs to compare the Komogorov rate to the rate at which particles collide at the pipe wall given by Equation 15. For an impaction velocity $\left|\mathbf{v}_{I}\right| \sim 10 \mathrm{~m} / \mathrm{s}$ and a Kolmogorov time scale $\tau_{K} \sim 10^{-5} s$ at the wall, the ratio of the Kolmogorov frequency over the impaction rate is $\sim 46$. From this result, the break-up due to turbulence is the dominant mechanism.

Another way to view the problem is to determine whether a particles travelling in the radial direction is likely to break-up in the bulk before reaching the wall.

The distance travelled by a fluid particle in the radial direction is given by:

$$
x=\sqrt{2 D_{T} \tau_{K}}
$$

where $D_{T}$ is the turbulent diffusion coefficient.

$$
D_{T}=0.1 d \overline{u_{f}^{\prime}}
$$

with the fluctuating part of the fluid velocity $u_{f}^{\prime} \sim 0.1 u_{f}$. For homogeneous and isotropic turbulence and particles in local equilibrium with the fluid turbulence:

$$
\frac{\overline{V_{p}^{\prime}}}{\overline{\overline{u_{f}^{\prime}}}}=\frac{1}{S t+1}
$$

The distance travelled by the particle in the radial direction is therefore given by:

$$
x_{p}=\frac{x}{S t+1},
$$

with the Stokes number $S t=\frac{\tau_{p}}{\tau_{K}}$ and $\tau_{p}$ the particle response time.

For $\rho_{p}=4.10^{3} \mathrm{~kg} / \mathrm{m}^{3} ; d_{p}=5 \mu \mathrm{m} ; \mu=1.98 .10^{-5} \mathrm{~kg} \cdot \mathrm{m}^{-1} \mathrm{~s}^{-1} ; u_{f} \sim 200 \mathrm{~m} / \mathrm{s}$, it is readily shown that the Stokes number $\mathrm{St} \sim 3$ and $D / x_{p} \sim 24$ with $D$ the diameter of the pipe. Therefore, it is assumed that agglomerates break-up before reaching the wall as the particle is likely to remain in the bulk. Thus, only break-up due to the turbulent shear stresses will be considered in the rest of this paper.

The abrupt expansion of the jet starting at the tube outlet (representing the tube rupture) results in a steep increase in the flow characteristics values. For example, the dissipation rate of turbulent kinetic energy as a function of downstream distance for different radial distances has been calculated using FLUENT 6.3 as shown in Fig. 10.

The dissipation rate profile identifies two regions which contribute to the particle break-up: (i) the pipe region where the agglomerates are under the action of quasi constant turbulent shear stresses function of an average value of the turbulent 
Fig. 10 dissipation rate for turbulent energy along the axis for a mass flow rate of $320 \mathrm{~kg} / \mathrm{h}$ in the $20 \mathrm{~cm}$ long tube. The centreline is denoted by $\mathrm{y}=0$ and the wall by $\mathrm{y}=8.3 \mathrm{~mm}$. The tube orifice is located at $\mathrm{x}=0$

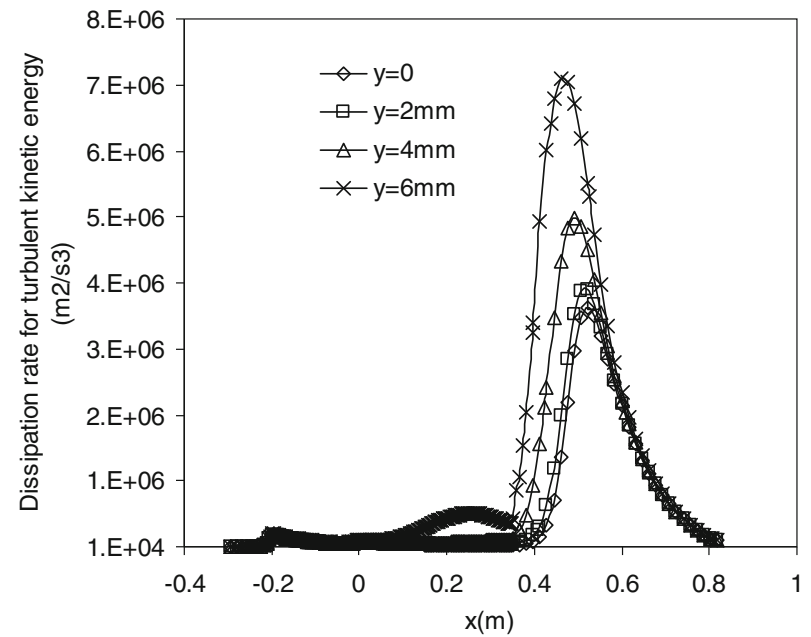

shear rate $\overline{\dot{\gamma}}$ and (ii) the turbulent jet region where agglomerates are exposed to a maximum value for the turbulent shear stresses as a function of the maximum turbulent shear rate in the turbulent free jet $\dot{\gamma}_{\max }$.

The maximum turbulent shear rate computed within FLUENT 6.3 is related to the measurement of the particle count median diameter taken after the tube in the conical expansion outlet. Figure 11 shows that a power law describes well the evolution of the particle count diameter as a function of the maximum turbulent shear rate. It is given by:

$$
d_{50 \%}=5.4 \times 10^{2} \dot{\gamma}_{\max }^{-m}
$$

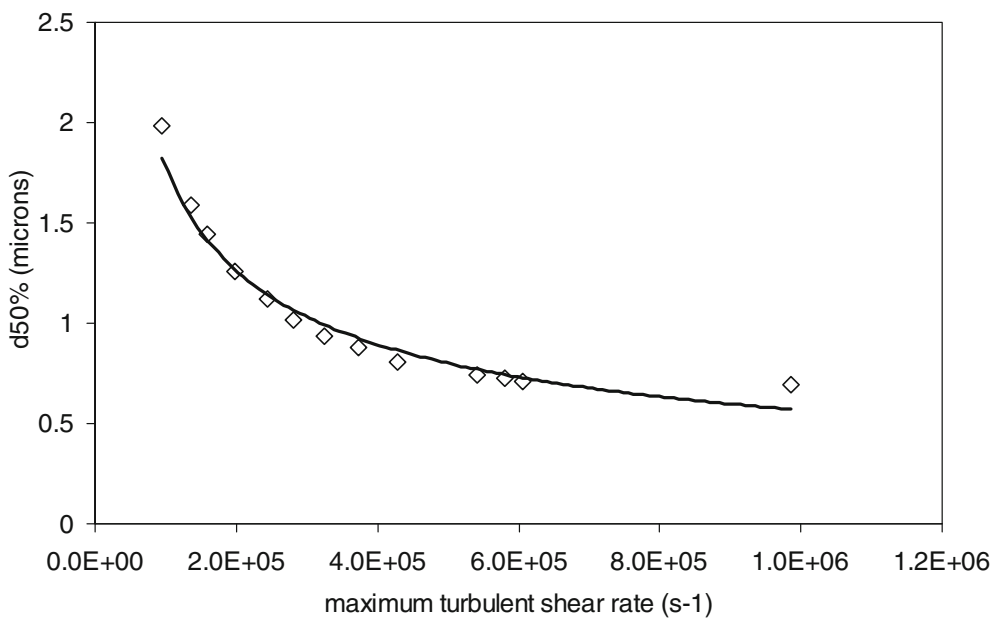

Fig. 11 Particle count diameter d50\% versus maximum Turbulent Energy Dissipation rate. The solid line represents the power law fit 
with $d_{50 \%}$ the particle count median diameter, $\dot{\gamma}_{\max }$ the maximum turbulent shear rate and $m=0.5$ a constant.

Equation 20 confirms that larger agglomerates break more rapidly and more easily than smaller ones which need more turbulent energy to break up.

\section{Particle Transport Model}

In the Lagrangian description, a fluid particle is defined as a point moving with the local fluid velocity. The trajectory of a fluid particle in $\mathrm{x}-t$ space is therefore given by time integration of

$$
\frac{\mathrm{D} \mathbf{x}}{\mathrm{D} t}=\mathbf{u}(\mathbf{x}, t)
$$

where the material derivative is defined by

$$
\frac{\mathrm{D}}{\mathrm{D} t} \equiv \frac{\partial}{\partial t}+U_{f_{i}} \frac{\partial}{\partial x_{i}}
$$

After solving the fluid dynamic equations, it is possible to export from FLUENT the values of the fluid properties along the trajectories of a fluid particle across the domain together with the Lagrangian time.

If the diffusive transport is neglected with respect to the convective transport then the break-up equation becomes

$$
\frac{\mathrm{D}}{\mathrm{D} t} n(L, t)=\int_{L}^{\infty} a^{\prime}(l, t) b^{\prime}(L, l) n(l, t) d l-a^{\prime}(L, t) n(L, t)
$$

Eq. 20 gives a power law for the evolution of $\mathrm{d}_{50 \%}$ as a function of the turbulent shear rate in the turbulent jet, so that the break-up rate is also assumed to follow a power law of the turbulent shear rate and the size such as: $a^{\prime}(L, t)=P_{1} \overline{\dot{\gamma}}^{P_{2}} L^{P_{3}}$ as given by Eq. 4 with $\bar{\gamma}$ a function of the spatial location $x$.

The minimum CMD observed experimentally was $0.6 \mu \mathrm{m}$. For the observed threshold size, the adhesive forces are therefore supposed to become greater than the aerodynamic forces due to the turbulent shear stresses at the surfaces of the agglomerates so that no break-up occurs for particle size below this threshold. Lognormal distribution has been observed as the most important class occurring in nature [56]. The break-up process can be interpreted as a random process obeying the law of proportionate effect [57]:

"A variate subject to a process of change is said to obey the law of proportionate effect if the change in the variate at any step of the process is a random proportion of the previous value of the variate."

The final particle size distribution being lognormal, the fragment distribution $b^{\prime}$ is expressed in terms of volume as:

$$
b^{\prime}(v, \varepsilon)=\delta\left(v-\left[1 /\left(1+c_{m}\right)\right] \varepsilon\right)+\delta\left(v-\left[c_{m} /\left(c_{m}+1\right)\right] \varepsilon\right),
$$


with $c_{m}$ being the mass ratio. If the instantaneous mass ratio is assumed to be selected from a uniform distribution of width $1, c_{m}$ referring to the mean value of the mass ratio is chosen to be 0.5 .

If the fragment distribution is replaced by Eq. 24, Eq. 23 can be expressed as

$$
\frac{\mathrm{D}}{\mathrm{D} t} n(v, \mathbf{x}, t)=S\left(v, v_{1}, v_{2}, \mathbf{x}, t\right)
$$

with

$$
\begin{aligned}
S= & {\left[\left(c_{m}+1\right) / c_{m}\right] a^{\prime}\left(v_{1}, \mathbf{x}, t\right) b^{\prime}\left(v_{1}, \varepsilon\right) n\left(v_{1} ; \mathbf{x}, t\right) } \\
& +\left(c_{m}+1\right) a^{\prime}\left(v_{2}, \mathbf{x}, t\right) b^{\prime}\left(v_{2}, \varepsilon\right) n\left(v_{2} ; \mathbf{x}, t\right)-a^{\prime}(v, \mathbf{x}, t) n(v, \mathbf{x}, t)
\end{aligned}
$$

And

$$
\begin{aligned}
& v_{1}=\left[\left(c_{m}+1\right) / c_{m}\right] v \\
& v_{2}=\left(c_{m}+1\right) v
\end{aligned}
$$

This type of equation can be readily solved by dividing the size domain into $m$ sections $\left[v_{h}-1, v_{h}\right], h=1,2, . ., m$. Equation 25 is integrated between $v_{h}-1$ and $v_{h}$.

The number density of particles belonging to a section becomes:

$$
N_{h}=\int_{v_{h-1}}^{v_{h}} n(v, t) d v
$$

In each section $h$, a representative particle is chosen with volume $v_{h}^{*}\left(v_{h-1} \leq v_{h}^{*} \leq v_{h}\right)$. It is assumed that each particle in the section behaves as such a particle and that the density function is constant in each section equal to $n\left(v_{h}^{*}, t\right)$ so that:

$$
N_{h}=\left(v_{h}-v_{h-1}\right) n\left(v_{h}^{*}, t\right)
$$

\section{Results}

The method of integration along streamlines is only applicable if the turbulent diffusion is negligible in front of convection. A preliminary result consists in determining that this is actually the case within the context of this work.

The radial turbulent diffusion towards the pipe walls can be quantified by calculating the characteristic time for diffusion deposition [58]:

$$
\tau_{\text {dif }}=D /\left(4 v_{\text {dif }}\right),
$$

with the diffusion velocity $v_{\text {dif }}=S h D_{T} / D, D$ being the diameter of the tube, $S h=$ $0.042 \operatorname{Re} f_{f}^{0.5} S c^{1 / 3}$ the turbulent Sherwood number, $S c=v / D_{T}$ the Schmidt number

Table 2 Parameters for simulation

\begin{tabular}{lll}
\hline $\mathrm{P}_{1}$ & $\mathrm{P}_{2}$ & $\mathrm{P}_{3}$ \\
\hline 0.018 & 0.75 & 0.5 \\
\hline
\end{tabular}


Fig. 12 Comparison of $L_{10}$ and $L_{32}$ obtained from GDE integration along a streamline and experiments for $2 \mathrm{~m}$ long tube at the outlet of the test section (one point at the exit of the expansion zone)

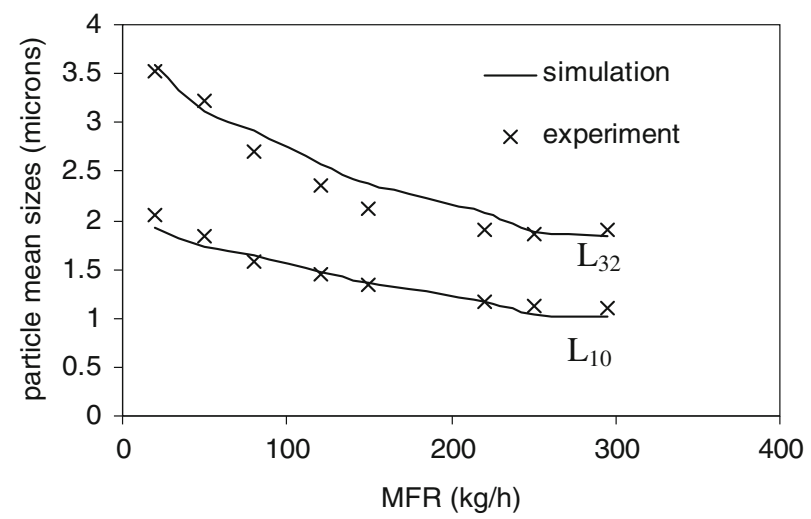

and $f_{f}$ the Fanning friction factor calculated with the Churchill expression such as [58]:

$$
\frac{2}{f_{f}}=\left\{\left[(8 / \operatorname{Re})^{10}+(\operatorname{Re} / 36500)^{20}\right]^{-1 / 2}+[2.21 . \ln (\operatorname{Re} / 7)]^{10}\right\}^{1 / 5}
$$

The characteristic time scale for diffusion is to be compared with the residence time, $\tau_{\text {res }}=D / U_{m}$, where $D$ is the tube diameter and $U_{m}$ is the axial mean velocity. The order of magnitude of these two time scales is summarized in Table 2.

The characteristic time for radial diffusion was found to be between $10^{-6} \mathrm{~s}$ and $10^{-4} \mathrm{~s}$ whereas the residence time was between $10^{-8} \mathrm{~s}$ and $10^{-6} \mathrm{~s}$ at the test section outlet. Furthermore, with reference to [6], the Peclet number is very large in zones of high turbulent shear stresses, where break-up is likely to occur. Therefore it can be assumed that convection is the dominant transport mechanism. For inertialess particles and in absence of diffusion the particles follow the streamlines in that their convective velocity becomes the local fluid velocity and therefore Eq. 25 applies.

The mean diameter $L_{10}=m_{1} / m_{0}$ and the Sauter diameter $L_{32}=m_{3} / m_{2}$ are computed for comparison with the experiments. The one dimensional GDE integration along a streamline is an efficient and simple procedure for analysing and interpreting

Fig. 13 Comparison of $L_{10}$ and $L_{32}$ obtained from GDE integration along a streamline and experiments for $4 \mathrm{~m}$ long tube at the outlet of the test section

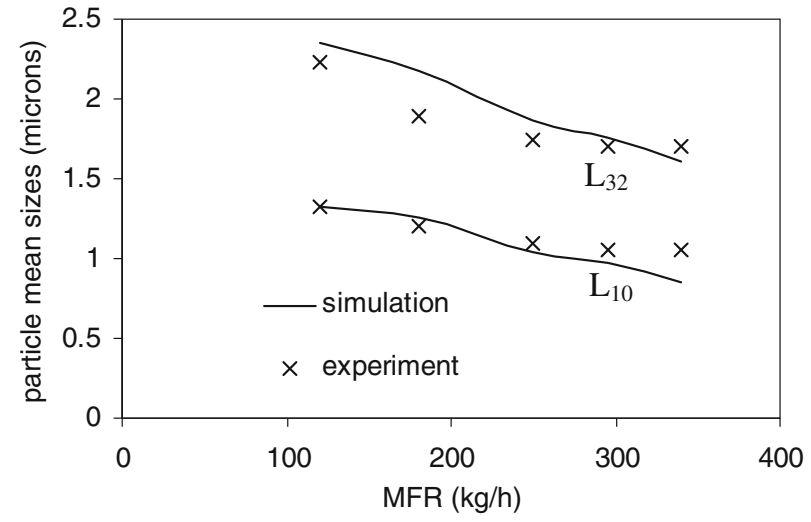


Table 3 Characteristic time scales for convection and radial diffusion

Fig. 14 Distribution function for the number density as a function of the sizeComparison between experiments and simulation for the PSD at the outlet. MFR $120 \mathrm{~kg} / \mathrm{h}-20 \mathrm{~cm}$ long tube

\begin{tabular}{llll}
\hline Pipe length $(\mathrm{m})$ & MFR $(\mathrm{kg} / \mathrm{h})$ & $\tau_{\text {dif }}(\mathrm{s})$ & $\tau_{\text {res }}(\mathrm{s})$ \\
\hline 0.2 & 120 & $10^{-6}-10^{-4}$ & $10^{-8}-10^{-7}$ \\
& 403 & $10^{-6}-10^{-4}$ & $10^{-8}-10^{-7}$ \\
2 & 80 & $10^{-6}-10^{-4}$ & $10^{-8}-10^{-7}$ \\
& 320 & $10^{-5}-10^{-4}$ & $10^{-8}-10^{-6}$ \\
\hline
\end{tabular}

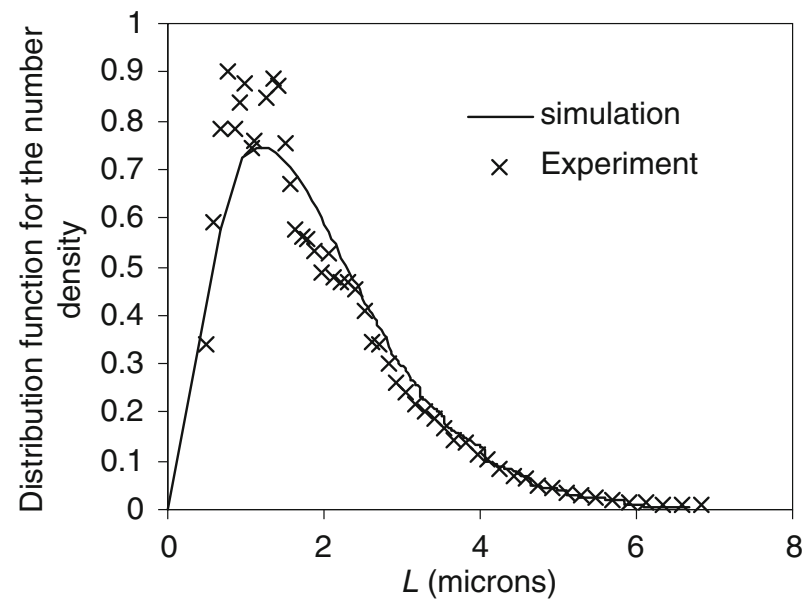

Fig. 15 Distribution function for the number density as a function of the size for different inlet mass flow rates- $(20 \mathrm{~cm}$ long tube test section)

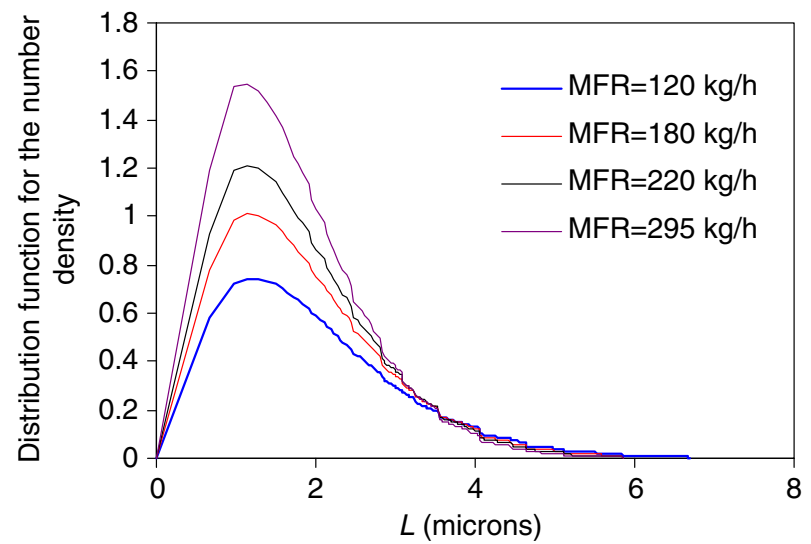


the experimental results under different levels of turbulence with good comparison with experimental results as shown in Figs. 12 and 13 which show the values of the moment ratio for simulation with the set of parameters given in Table 3 and for experimental results.

The simulation reproduces very well the tail of the lognormal distribution, see Fig. 14. The model over-estimates the variance of the distribution with a maximum difference of $15 \%$ which is acceptable considering an uncertainty on the measurement of about $7 \%$ [6].

As expected, the area under the curves increases with the level of turbulence since the number of particles resulting from the break-up process inevitably increases in absence of agglomeration, see Fig. 15.

Fig. 16 a. Evolution of the distribution function for the PSD along the pipe axis (x coordinate) of the $2 \mathrm{~m}$ long pipe test section. b. Comparison of the simulated distribution at the exit of the pipes ( $2 \mathrm{~m}$ and $4 \mathrm{~m}$ length) and downstream at the exit of the expansion zone (2.5 $\mathrm{m}$ and $4.5 \mathrm{~m}$ )

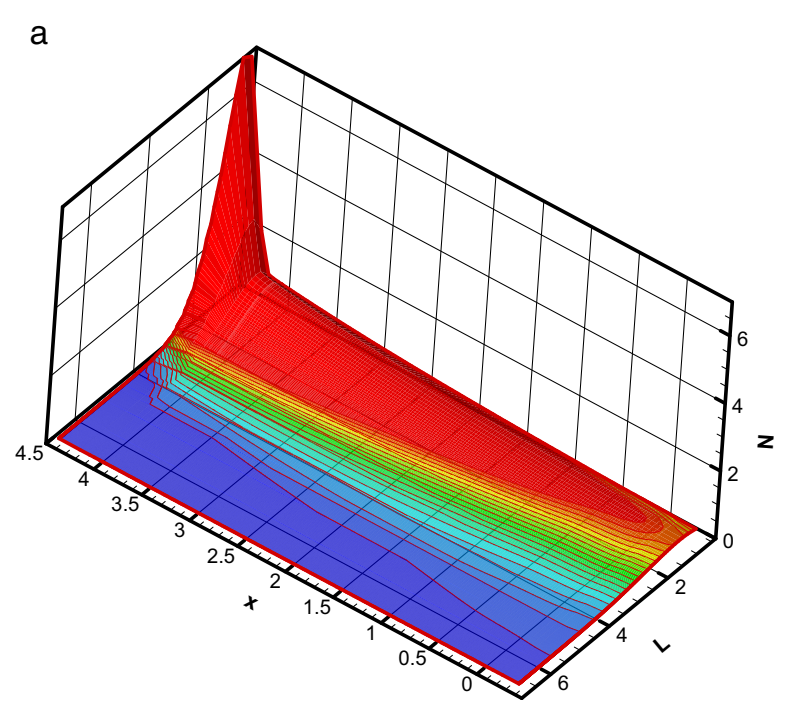

b

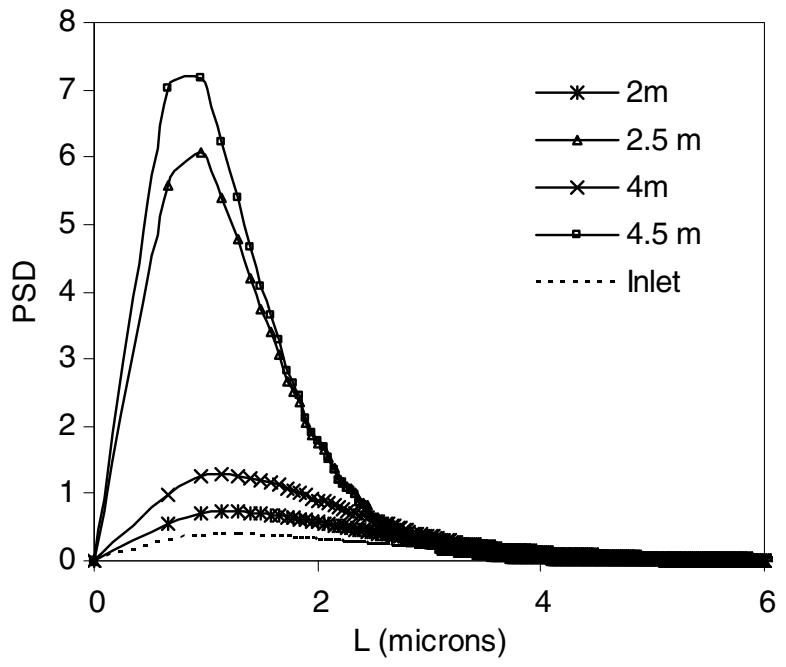


Figure 16a gives the PSD change along the axis for $4 \mathrm{~m}$ long tube and Fig. 16b compares the PSD just at the exit of the pipe ( $2 \mathrm{~m}$ and $4 \mathrm{~m}$ locations $)$ and the PSD at the exit of the expansion zone $(2.5 \mathrm{~m}$ and $4.5 \mathrm{~m}$ locations $)$ with the distribution measured at the inlet of the test section. In the expansion zone, the area under the PSD curve increases sharply which reveals an important increase in the number of particles in the domain due to break-up. The two regions of break-up identified earlier appear quite clearly i.e. break-up due to constant turbulent shear stresses in the pipe and break-up under the action of very high local shear stresses in the expansion zone. Furthermore, from the results illustrated in Fig. 16, it now appears clearly that the expansion zone is the zone where most of the break-up takes place.

\section{Discussion}

The turbulent flow field simulated with CFD was validated against pressure and LDA measurements and it was used to determine the magnitude of the parameters relevant for the break-up, as well as the associated exposure times. Break-up of particles by turbulent stresses was identified to be the dominant mechanism responsible for the observed reduction in particle size following a tube break. Also, for particles smaller than the Kolmogorov length scale, turbulent stresses which acted on the particles were shown to be shear stresses due to the instantaneous fluid velocity difference across the agglomerate diameter. The turbulent shear rate, which was a function of the turbulent dissipation rate and the kinematic viscosity, was the most relevant parameter to be taken into account for the break-up process, together with the residence time of the particles in the tube. It is worth mentioning that the fluctuations of the turbulent shear rate resulting in fluctuations of the shear stresses acting on the aggregates were not considered.

The near wall region did not affect break-up since the break-up of particles by impaction at the wall was shown to be negligible for $\mathrm{T}_{\mathrm{i}} \mathrm{O}_{2}$ agglomerates in the range $0.1 \mu \mathrm{m}-5 \mu \mathrm{m}$. Furthermore, attaining sonic conditions was shown not to play a role in particle break-up and therefore there existed no extra force acting on the particles compared to the case of particle-laden incompressible turbulent flows.

The latter conclusions about break-up mechanisms were used to define the appropriate source terms in the Population Balance equation. The radial and axial diffusive transport could be neglected with respect to the convective transport in the regions where the break-up took place and therefore, the GDE could be integrated along streamlines.

The effect of particle inertia on transport can be estimated via the Stokes number. For a Kolmogorov time scale $\sim 10^{-5} \mathrm{~s}$ the Stokes number is $S t \sim 10^{-1}$ for a typical agglomerate size of $1 \mu \mathrm{m}$ and $S t \sim 3$ for $5 \mu \mathrm{m}$. This suggests that for the largest particles considered in this work, the inertia of the particles could play a role in the calculation of the forces acting on the particles.

Although it was only exact for inertialess particles in the bulk, the model given here reproduced very well the experimental data trend. In addition, it offered a computationally efficient approach (when compared with solving the full population balance), allowed the use of as many as 500 size sections to discretise the particle size distribution and thereby provided directly the evolution of the distribution along the pipe length, instead of using closure approximation model based on the moments of the distribution. The simulation results agreed with the experimental analysis. 


\section{Conclusion}

The break-up of agglomerates in very high turbulent pipe flows has been analysed. Due to the complexity of the flow field, CFD was used to examine the relevant mechanisms responsible for the break-up. It was found that under the investigated conditions, the break-up was dominated by that due to turbulent shear stresses.

The proposed one dimensional model consisting of integrating the GDE along streamlines was validated against experimental data. It is a computationally efficient approach which was shown to describe well the changes in the distribution of aerosol agglomerates subjected to highly turbulent flow whilst not accounting for the influence of the fluctuations of the turbulent stresses and the inertial effect which can be important for particle sizes greater than one micron.

Two regions of break-up were identified. In the pipe, the particles see continuously constant turbulent shear stresses and break-up to a lesser extent than in the expansion zone where particles break-up instantaneously under the action of very high local shear stresses.

Acknowledgements The authors would like to thank PSI for funding this research programme and for carrying out the experimental tests. The technical support from Dr Daniele Marchisio and Dr Ryan Sidin is also gratefully acknowledged.

\section{References}

1. Falkovich, G., Fouxon, A., Stepanov, M.G.: Acceleration of rain initiation by cloud turbulence. Nature 419, 151-154 (2002)

2. Kusters, K.A.: The influence of turbulence on aggregation of small particles in agitated vessel. $\mathrm{PhD}$ thesis. Eindhoven University of Technology, The Netherlands (1991)

3. Marchisio, D., Vigil, D., Fox, R.: Implementation of the quadrature method of moments in CFD codes for aggregation-breakage problems. Chem. Eng. Sci. 58(15), 3337-3351 (2003)

4. Voss, A., Finlay, W.H.: Deagglomeration of dry powder pharmaceutical aerosols. Int. J. Pharm. 248(1-2), 39-50 (2002)

5. Lasheras, J.C., Eastwood, C., Martinez-Bazan, C., Montanes, J.L.: A review of statistical models for the break-up of an immiscible fluid immersed into a fully developed turbulent flow. Int. J. Multiphas. Flow 28(2), 247-278 (2002)

6. Ammar, Y.: Turbulent agglomeration and break-up of nuclear aerosols. $\mathrm{PhD}$ thesis. Newcastle University, UK (2008)

7. Lind, T., Ammar, Y., Dehbi, A., Guentay, S.: De-agglomeration mechanisms of TiO2 aerosol agglomerates in PWR steam generator tube rupture conditions. Nucl. Eng. Des. 240(8), 2046$2053(2010)$

8. Sonntag, R.C., Russel, W.B.: Structure and breakage of flocs subjected to fluid stresses, I. Shear experiments. J Colloid Interface Sci 113, 399-413 (1986)

9. Pietsch, W.: Agglomeration Processes: Phenomena, Technologies, Equipment. Wiley-VCH, Weinheim (2002)

10. Ramkrishna, D.: Population Balances: Theory and Applications to Particular Systems in Engineering. Academic Press, San Diego (2000)

11. Kramer, T.A., Clark, M.M.: Incorporation of aggregate breakup in the simulation of orthokinetic coagulation. J. Colloid Interface Sci. 216(1), 116-126 (1999)

12. Luo, H., Svendsen, H.F.: Theoretical model for drop and bubble breakup in turbulent dispersions. AIChE J. 42(5), 1225-1233 (1996)

13. Coulaloglou, C.A., Tavlarides, L.L.: Description of interaction processes in agitated liquid-liquid dispersions. Chem. Eng. Sci. 32(11), 1289-1297 (1977)

14. Tambo, N., Watanabe, Y.: Physical aspect of flocculation process - I: Fundamental treatise. Water Res. 13(5), 429-439 (1979) 
15. Potanin, A.A.: On the computer simulation of the deformation and breakup of colloidal aggregates in shear flow. J. Colloid Interface Sci. 157(2), 399-410 (1993)

16. Babler, M.U., Morbidelli, M., Baldyga, J.: Modelling the breakup of solid aggregates in turbulent flows. J. Fluid Mech. 612, 261-289 (2008)

17. Fair, G.M., Gemmell, R.S.: Mathematical model of coagulation. J. Coll. Sci. 19, 360-372 (1964)

18. Grabenbauer, G.C., Glatz, C.E.: Protein precipitation-analysis of particle size distribution and kinetics. Chem. Eng. Commun. 12(1), 203-219 (1981)

19. Shiga, S., Furuta, F.: Processibility of EPR in an internal mixer (II)-morphological changes of carbon black agglomerate during mixing. Rubber Chem. Technol. 58(1), 1-22 (1985)

20. Valentas, K.J., Amundson, N.R.: Breakage and coalescence in dispersed phase systems. Ind. Eng. Chem. Fundam. 5(4), 533-542 (1966)

21. Collins, S.B., Knudsen, J.G.: Drop-size distributions produced by turbulen pipe flow of immiscible liquids. AIChE J. 16(6), 1072-1080 (1970)

22. Chatzi, E.G., Gavrielides, A.D., Kiparissides, C.: Generalized model for prediction of the steadystate drop size distributions in batch stirred vessels. Ind. Eng. Chem. Res. 28(11), 1704-1711 (1989)

23. Peng, S.J., Williams, R.A.: Direct measurement of floc breakage in flowing suspensions. J. Colloid Interface Sci. 166(2), 321-332 (1994)

24. Kumar, S., Kumar, R., Gandhi, K.S.: Alternative mechanisms of drop breakage in stirred vessels. Chem. Eng. Sci. 46(10), 2483-2489 (1991)

25. Hesketh, R.P., Etchells, A.W., Russell, T.W.F.: Bubble breakage in pipeline flow. Chem. Eng. Sci. 46(1), 1-9 (1991)

26. Tsouris, C., Tavlarides, L.L.: Breakage and coalescence models for drops in turbulent dispersions. AIChE J. 40(3), 395-406 (1994)

27. Kostoglou, M., Dovas, S., Karabelas, A.J.: On the steady-state size distribution of dispersions in breakage processes. Chem. Eng. Sci. 52(8), 1285-1299 (1997)

28. Diemer, R.B., Olson, J.H.: A moment methodology for coagulation and breakage problems: part 3-generalized daughter distribution functions. Chem. Eng. Sci. 57(19), 4187-4198 (2002)

29. Peterson, T.W.: Similarity solutions for the population balance equation describing particle fragmentation. Aerosol Sci. Tech. 5(1), 93-101 (1986)

30. Ziff, R.M.: New solutions to the fragmentation equation. J. Phys. A- Math. Gen. 24(12), 28212828 (1991)

31. Diemer, J.B.R., Olson, J.H.: A moment methodology for coagulation and breakage problems: part 1-analytical solution of the steady-state population balance. Chem. Eng. Sci. 57(12), 21932209 (2002)

32. Diemer, J.R.B., Spahr, D.E., Olson, J.H., Magan, R.V.: Interpretation of size reduction data via moment models. Powder Technol. 156(2-3), 83-94 (2005)

33. Smith, M., Matsoukas, T.: Constant-number Monte Carlo simulation of population balances. Chem. Eng. Sci. 53(9), 1777-1786 (1998)

34. Kumar, S., Ramkrishna, D.: On the solution of population balance equations by discretization-I. A fixed pivot technique. Chem. Eng. Sci. 51(8), 1311-1332 (1996)

35. Hill, P.J., Ng, K.M.: New discretization procedure for the agglomeration equation. AIChE J. 42(3), 727-741 (1996)

36. Vanni, M.: Discretization procedure for the breakage equation. AIChE J. 45(4), 916-919 (1999)

37. McGraw, R.: Description of aerosol dynamics by the quadrature method of moments. Aerosol Sci. Tech 27(2), 255-265 (1996)

38. Bakhtar, F., Mohammadi, T.M.T.: An investigation of two-dimensional flows of nucleating and wet steam by the time-marching method. Int. J. Heat Fluid Flow 2(1), 5-18 (1980)

39. Moheban, M., Young, J.B.: A study of thermal nonequilibrium effects in low-pressure wet-steam turbines using a blade-to-blade time-marching technique. Int. J. Heat Fluid Flow 6(4), 269-278 (1985)

40. White, A.J., Young, J.B.: Time-marching method for the prediction of two-dimensional, unsteadyflows of condensing steam. J. Propuls. Power 9(4), 579-587 (1993)

41. Skillings, S.A., Jackson, R.: A robust "time-marching" solver for one-dimensional nucleating steam flows. Int. J. Heat Fluid Flow 8(2), 139-144 (1987)

42. Young, J.B.: Two-dimensional nonequilibrium wet steam calculations for nozzles and turbine cascades. J. Turbomach. 114, 569 (1992) 
43. Hagmeijer, R., Ijzermans, R.H.A., Put, F.: Solution of the general dynamic equation along approximate fluid trajectories generated by the method of moments. Phys. Fluid 17(5), 056101$056112(2005)$

44. Wengeler, R., Nirschl, H.: Turbulent hydrodynamic stress induced dispersion and fragmentation of nanoscale agglomerates. J. Colloid Interface Sci. 306(2), 262-273 (2007)

45. Zumaeta, N., Cartland-Glover, G.M., Heffernan, S.P., Byrne, E.P., Fitzpatrick, J.J.: Breakage model development and application with CFD for predicting breakage of whey protein precipitate particles. Chem. Eng. Sci. 60(13), 3443-3452 (2005)

46. Iliopoulos, I., Hanratty, T.J.: Turbulent dispersion in a non-homogeneous field. J. Fluid Mech. 392, 45-71 (1999)

47. Dehbi, A.: A CFD model for particle dispersion in turbulent boundary layer flows. Nucl. Eng. Des. 238(3), 707-715 (2008)

48. Soldati, A.: Particles turbulence interactions in boundary layers. ZAMM - J. Appl. Math. Mech. / Zeitschrift für Angewandte Mathematik und Mechanik 85(10), 683-699 (2005)

49. Marchisio, D.L., Soos, M., Sefcik, J., Morbidelli, M.: Role of turbulent shear rate distribution in aggregation and breakage processes. AIChE J. 52(1), 158-173 (2006)

50. Abrahamson, J.: Collision rates of small particles in a vigorously turbulent fluid. Chem. Eng. Sci. 30, 1371-1379 (1975)

51. Saffman, P.G., Turner, J.S.: On the collision of drops in turbulent clouds. J. Fluid Mech. 1(01), 16-30 (1956)

52. Tennekees, H., Lumley, J.L.: A First Course in Turbulence. The Massachusetts Institute of Technology, US (1972)

53. Dreeben, T.D., Pope, S.B.: Probability density function/Monte Carlo simulation of near-wall turbulent flows. J. Fluid Mech. 357, 141-166 (1998)

54. Antonia, R.A., Teitel, M., Kim, J., Browne, L.W.B.: Low-Reynolds-number effects in a fully developed turbulent channel flow. J. Fluid Mech. 236, 579-605 (1992)

55. Fan, L.S. and Zhu, C.: Principles of Gas-Solid Flows: Cambridge Series in Chemical Engineering. Cambridge University Press, UK (1998)

56. Kapteyn, J.C.: Skew frequency curves in biology and statistics. Mol. Gen. Genet. 19(3), 205-206 (1918)

57. Aitchison, J., Brown, J.A.C.: The Lognormal Distribution. University Press, UK (1963)

58. Housiadas, C., Drossinos, Y.: Thermophoretic deposition in tube flow. Aerosol Sci. Tech 39(4), 304-318 (2005) 